\title{
Medical versus surgical treatment of stable angina pectoris: progress report of a large scale study
}

\author{
Herbert N. Hultgren \\ M.D. \\ TIMOTHY TAKARO \\ M.D. \\ KATHERINe Detre \\ M.D. \\ and

\section{Participants in the Veterans Administration Cooperative Study}

\author{
Cardiology Service, Palo Alto Veterans Administration Hospital, Palo Alto, California and the \\ Asheville Veterans Administration Hospital, Oteen, North Carolina, U.S.A.
}

\begin{abstract}
Summary
A large scale, prospective, randomized study of surgical $v$. medical management of disabling angina pectoris is being conducted as a cooperative study among thirteen Veterans Administration hospitals in the U.S.A. A total of 1015 patients have been entered into the study and follow-up data are currently being evaluated. Patient entry into the study was concluded in December 1974. Patient compliance has been acceptable with only $7 \%$ of patients not adhering to their randomization category. Thirty-day operative mortality (1972-1974) in 309 patients was 5.3\%. The patient population exhibited a severe degree of coronary disease. There was ECG evidence of prior myocardial infarction in $\mathbf{4 0} \%$. There were significant obstructive lesions in three major coronary arteries in $51 \%$ and significant lesions of the left main coronary artery in $11 \%$. Medical and surgical treatment groups demonstrated no significant differences in objective descriptive characteristics. Mortality in the medical group at 1 year was $8 \%$. Mortality was influenced by several factors including the number of vessels involved, left ventricular function and the presence of left main coronary artery disease. The lowest mortality occurred in patients with single vessel disease and normal LV function who had a 1-year mortality of $3 \%$. Patients with 3-vessel disease and abnormal LV function exhibited a 14\% 1-year mortality. Patients with disease of the left main coronary artery and poor LV function had a 1-year mortality of $37 \%$. Analyses of the results of treatment modalities in sub-groups is currently being performed and will be reported in future publications.
\end{abstract}

Supported by the Veterans Administration Cooperative Study.

Address for reprints: Herbert N. Hultgren, Veterans Administration Hospital, 3801 Miranda Avenue, Palo Alto, California 94304, U.S.A.
Introduction

Since the initial experimental work of Claude Beck many surgical procedures have been designed for the symptomatic relief of angina pectoris (Hultgren and Hurley, 1968). Most of these operations involved attempts to provide extra-cardiac sources of myocardial blood flow to the ischaemic left ventricle and included pericardial scarification, application of pedicles of omentum to the surface of the heart, internal mammary artery ligation and direct implantation of the internal mammary artery into the myocardium (Vineberg, 1964).

In the early 1960s, the internal mammary implant procedure was employed in an increasing number of surgical centres for several reasons: (1) experimental work by Vineberg had demonstrated that dogs with implants could survive coronary artery ligation without infarction or death (Vineberg, 1964); (2) angiography had demonstrated patency of implants in man and communications between the implanted artery and the native coronary circulation (Effler et al., 1963); (3) the operation could be performed with a low mortality and resulted in apparent symptomatic relief of angina (Effler et al., 1965) in many patients.

While the results appeared promising no systematic controlled trials had been carried out and it had been noted that earlier operations no longer performed by cardiac surgeons had been followed by similar apparent improvement in symptoms. There was no evidence that the operation improved longevity or prevented myocardial infarction.

For these reasons, the Veterans Administration initiated a co-operative study of the implant procedure in 1968. A prospective, randomized protocol was designed.

Patients with stable angina with arteriographic evidence of significant coronary artery disease were randomized into a medical and a surgical treatment group. Patients randomized to surgery had either 
single or double internal mammary artery implants. Endarterectomy, myocardial resection or valve replacement were not performed. After entry into the study or surgery, patients were seen at 3-month intervals. At 1 year, initial studies were repeated in all patients including shunt angiography in surgically treated patients. A total of 100 patients were randomized between 1968 and 1973.

In 1970 it became apparent that the saphenous vein bypass graft technique provided a superior method of revascularization. The previous protocol was accordingly modified to include an evaluation of the bypass procedure and since 1973 internal mammary implants were no longur performed on patients entered into the study. Long-term follow-up results of the implant patients have been completed and are described in a separate communication (Bhayana et al., 1975).

\section{Current protocol details}

\section{(A) Patient population}

Male patients with a history of stable angina of at least 6 months' duration who had been on acceptable medical treatment for 3 months were eligible for the study. Objective evidence of coronary artery disease as demonstrated by ECG signs of prior infarction, ischaemia or a positive exercise test was required. If the patient agreed to enter the study, coronary arteriography and LV angiography were performed. Obstructive coronary lesions reducing the diameter of the lumen by more than $50 \%$ were considered significant. Coronary vessels should be suitable for bypass grafting. Patients with left ventricular aneurysms were excluded. Additional exclusions were: (1) myocardial infarction within 6 months of entry; (2) persistent diastolic hypertension ( $>100 \mathrm{mmHg}$ ) not responsive to medical treatment; (3) marked cardiac enlargement or other significant cardiac disease such as valvular disease; (4) presence of other major disease which would be a contra-indication to cardiac surgery or limit expectation of life; (5) previous cardiac surgery; (6) persistent congestive failure not responding to appropriate medical therapy; (7) participation in other Veterans Association Cooperative studies.

These criteria conformed to generally acceptable guide-lines for selecting patients for surgery if the operation had already been proved to be superior to medical treatment.

\section{(B) Informed consent}

After preliminary non-invasive screening, eligible patients were informed of the general nature of the study as follows:

'Your heart condition after coronary arteriography may be treated by a surgical operation or by medical treatment. We do not at this time know with cer- tainty which is the better therapy. There is a very small element of risk in coronary arteriography, but $\Phi$ this is outweighed by the real benefit to you of an $\frac{\varrho}{c}$ accurate assessment of the status of your coronary vessels, even if this Study were not being conducted. $\stackrel{5}{\rightarrow}$ If, after your coronary arteriography, it is decidedo that you should have either surgery or medical therapy, would you be willing to return for follow-up $\frac{\bar{c}}{\frac{1}{\sigma}}$ studies at intervals of 3 months? If you have surgery, $\stackrel{\odot}{\varnothing}$ would you also be willing to have another angiogram $₫$ after 1 year to determine the patency of the graft?'

If the patient expresses his willingness to have? coronary arteriography and to participate in the $\overrightarrow{\vec{\omega}}$ study, including follow-up visits four times a year, $\stackrel{\circ}{\circ}$ he is accepted for arteriography and a consent form 8 is signed after a full description of the study.

The amount of information given to the patient $\mathrm{N}$ about how his therapy is decided on, depends on the physician's assessment of what is in the best interests $\vec{P}$ of his patient, and the level of understanding and $\mathrm{v}$ interest shown by the patient. For the intelligent, inquisitive patient, who does not exhibit great anxiety, 음 all the facts about the study may be explained, in- cluding the possible advantages and disadvantages ${ }_{\infty}$ of both medical and surgical therapy, our current $\varnothing$ lack of knowledge about which treatment is moke 3 appropriate, and the fact that the decision will made randomly. For patients with less interest in the $\overrightarrow{0}$ study, or greater anxiety, the advantages and dis advantages of both modes of treatment are describ and the patient is told that his treatment will be selected after all his clinical and laboratory data have been reviewed.

The patient is reassured that he has the right to $\stackrel{\circ}{\frac{0}{2}}$ decline to participate in the study or to drop out of $\stackrel{\Phi}{\varrho}$ the study at any time without any jeopardy to his $\overrightarrow{\overrightarrow{0}}$ continued care. The patient is informed that his 3 eligibility to enter the study will be determined by highly trained physicians who will review all available information regarding his disease. He is also in- $\bar{\partial}$ formed that only his assignment to a treatment group will be done by randomization.

When the patient's consent to enter the study has been obtained angiography is performed and all data are reviewed by the cardiologist, angiographer and cardiac surgeon to determine if operable lesions are present and if the patient is suitable for entry into the $D$ study.

\section{(C) Randomization}

Patients who are eligible for the study are interviewed again to determine their willingness to accept either medical or surgical therapy.

Randomization is performed either at the participating hospital or the central co-ordinating centre. Envelopes for treatment assignments are prepared in advance from twenty-four lists of random permuta- 
tions in groups of ten. The number of medical and surgical assignments is balanced within each group but the sequence of assignments is different in each of the twenty-four groups.

The following stratification groups were separated: (1) age under 50 years and age over 50 years; (2) angiographic evidence of both anterior and inferior ischaemia; (3) anterior ischaemia only or posterior ischaemia only.

Patients randomized to surgery are scheduled for operation as soon as possible. Approximately $65 \%$ of patients had surgery performed within 30 days of randomization.

The starting time for the evaluation of treatment was defined as the date of randomization.

\section{(D) Screening records}

It is important in any study of treatment methods to obtain accurate information about the population group from which study patients are selected. For this reason, two screening records are maintained by each participating hospital. All patients initially considered eligible for the study before coronary arteriography are entered into an initial screen record. After the completion of non-invasive studies, patients who are considered ineligible for the study are identified in the record and the reasons for rejection are noted. Frequent reasons for rejection at this point were: (1) insufficient angina; (2) absence of ECG evidence of coronary disease; (3) presence of other disease. All patients who have angiography are entered into a second screening record and reasons for rejection of ineligible patients are indicated. Fre-

TABLE 1. Reasons for rejection on initial screening

\begin{tabular}{lr}
\hline \multicolumn{1}{c}{ Reason } & \multicolumn{1}{c}{$\%$} \\
\hline Insurticient history of angina & $13 \cdot 4$ \\
Does not meet ECG criteria & $9 \cdot 0$ \\
Other disease & $8 \cdot 2$ \\
Myocardial infarct in last 6 months & $7 \cdot 4$ \\
Unwilling & $4 \cdot 5$ \\
Left ventricular failure & $3 \cdot 6$ \\
Unreliable & $2 \cdot 5$ \\
Previous cardiac surgery & $2 \cdot 1$ \\
\hline
\end{tabular}

TABLE 2. Reasons for rejection following coronary arteriography

\begin{tabular}{lr}
\hline \multicolumn{1}{c}{ Reason } & \multicolumn{1}{c}{$\%$} \\
\hline Insignuicant lesions & $1 \cdot \cdot 3$ \\
Ventricular aneurysm & $7 \cdot 4$ \\
Unwilling & $6 \cdot 8$ \\
Poor myocardial contractility & $6 \cdot 1$ \\
Insufficient symptoms & $3 \cdot 9$ \\
Death & $1 \cdot 5$ \\
Technically unsatisfactory arteriogram & $1 \cdot 2$ \\
Unreliable & $1 \cdot 1$ \\
\hline
\end{tabular}

quent reasons for rejection at this point were: (1) insufficient coronary disease; (2) left ventricular aneurysm; (3) unwilling to enter study. A summary of the number of patients screened and rejected is indicated in Tables 1 and 2 . Approximately $33 \%$ of patients initially entered had angiography and approximately $50 \%$ of those who had angiography entered the study.

\section{(E) Medical treatment}

Medical treatment consisted of the use of currently accepted methods for the relief of angina including nitrates, propranolol, correction of mild hypertension and the use of diuretics and digitalis when indicated. Auxiliary measures such as dietary modification of sodium intake, fat content as well as weight reduction were employed when necessary. A systematic required programme was not employed.

\section{(F) Surgical treatment}

Following surgery, no patient was denied any mode of medical treatment when appropriate indications were present. For this reason the study, in a sense, evaluates medical treatment alone $v$. surgical and medical treatment.

\section{(G) Follow-up studies}

Since the validity of any study of different treatment methods depends heavily on complete and accurate follow-up data, several methods were employed in the study to accomplish this objective:

(1) Follow-up forms are completed after every 3month visit for the first 2 years and after every 6month visit for the final 3 years. A copy of the ECG recorded at each visit is enclosed with the follow-up report and sent to the study centre.

(2) A report of studies performed annually after randomization is prepared and sent to the study centre.

(3) Special reports are also prepared on new admissions to hospital, and on death.

To ensure completeness of follow-up, each participating hospital is sent a monthly schedule of followup visits to be performed and any data points that are needed to complete all forms.

Delinquent follow-ups are traced to a central VA data file called BIRLS for survival information. If no data are available in BIRLS another VA central data bank is consulted (Patient Treatment File) regarding the patient's treatment at other VA hospitals. Only $1 \%$ of the patients in the current study have delinquent follow-up data. Complete follow-up information is especially important in any study where mortality is a primary end-point since a high percentage of patients who are lost to follow-up may be lost because of death. The resources of the VA 
system ensure that all deaths can be accurately traced.

\section{(H) Adherence to treatment groups}

Every reasonable effort is made during the screening period to select patients who are reliable and willing to accept the assigned treatment.

Approximately $7 \%$ of patients (thirty-five patients) assigned to surgery refused operation after randomization or died before surgery was performed (eight patients).

A similar percentage (thirty-five patients) in the group assigned to medical treatment subsequently had surgery either by their own choice or at the advice of a physician. Follow-up data are available on all patients who did not adhere to the assigned treatment group.

\section{(I) End-points of the study}

The primary end-point is overall mortality in both treatment groups.

Secondary end-points comprise an assessment of 'quality of life' and consist of the following: (1) nonfatal but disabling complications of surgery; (2) hospital admissions for cardiac reasons or late surgical complications; (3) non-fatal myocardial infarction including peri-operative infarction; (4) closure of vein bypass grafts as assessed by angiography; (5) severity of angina evaluated by a systematic, scorable questionnaire (Pfeifer, Hultgren and Fails, 1974); (6) exercise testing.

Less secure secondary end-points include: (1) New York Heart Association functional classification; (2) working status; (3) status of angina compared to entry data described as absent, improved, unchanged or worse.

\section{(J) Operational problems}

In the course of any long-term, large scale study of treatment methods certain unexpected operational problems arise which require modification of the protocol or appropriate solutions. Four such problems in the present study may be summarized:

(1) Changes in participating hospitals. Initially, twenty-one VA hospitals met predetermined qualification requirements and participated in the study. Subsequently, two additional hospitals were added and during the course of the study ten hospitals dropped out of the study. Changes in professional personnel, inadequate number of patients entered and a deterioration in quality of performance were the principal reasons for discontinuation of participation. Active hospitals and patient input are summarized in Table 3.

(2) Mortality of coronary arteriography. In 1972 it became apparent that the mortality and morbidity of coronary arteriography was unacceptably high. This
TABle 3. Patients randomized by participating hospitals

\begin{tabular}{lc}
\hline VA hospital & $\begin{array}{c}\text { No. of patients } \\
\text { randomized }\end{array}$ \\
\hline Little Rock & 153 \\
Hines & 148 \\
Palo Alto & 136 \\
Dallas & 120 \\
Buffalo & 85 \\
Madison & 74 \\
Oteen & 64 \\
Minneapolis & 60 \\
Cleveland & 50 \\
West Roxbury & 45 \\
Long Beach & 32 \\
Ann Arbor & 25 \\
Washington & 23 \\
Inactive hospitals & 26 \\
Total & 1041 \\
\hline
\end{tabular}

was not confined to the participant hospitals but was reported from other non-VA hospitals as well (Selzer, Anderson and March, 1971). A survey was conducted of the risks associated with arteriography in all participant hospitals and appropriate recommendations were made which have subsequently greatly reduced the risk of the procedure (Takaro et al., 1973). Pertinent data are summarized in Fig. Further to reduce risk coronary arteriography was n 8 longer necessary at the 1-year follow-up study bu became an optional procedure based on clear clinicat के indications.

(3) Operative mortality. In 1973 it was observed that some participating hospitals had an operative mortality which was substantially higher than other hospitals with a similar patient population. Visits were made to the appropriate hospitals and recommendations were made that would decrease mortality. As a result, operative mortality in three hospitals decreased substantially. Currently, the operative mortality in active hospitals from 1972 to 1974 in 309 patients is $5.3 \%$. The overall mortality is $8.8 \%$. Mortality is expressed as a 30 -day mortality to include late operative deaths. The operative mortality in the study is comparable to that reported from other

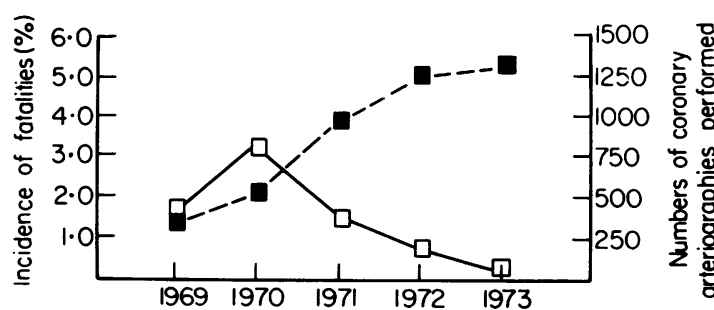

FIG. 1. Mortality from coronary arteriography in the VA Cooperative Study 1969-1973. $\ldots$, Volume of coronary arteriographies; $\square-\square$, incidence of fatalities. 
large non-VA hospitals (Table 4). The difference in mortality between the VA patients and the Stanford series (Table 4) may be related to the presence of more severe coronary disease in the VA study population which had a higher indicence of 3-vessel disease, i.e. $48 \%$ v. $38 \%$.

TABLE 4. Comparison of baseline variables in VA Cooperative Study patients (surgical) with Duke and Stanford series

\begin{tabular}{lccc}
\hline & VA Coop. & & \\
& Study & Duke & Stanford \\
\hline Number & 325 & 379 & 350 \\
Age mean & 50 & 51 & 52 \\
Angina > 5 years & $26 \%$ & $25 \%$ & $34 \%$ \\
ECG signs of prior infarct & $40 \%$ & $53 \%$ & $60 \%$ \\
3-Vessel disease & $48 \%$ & $48 \%$ & $38 \%$ \\
Class III \& IV NYHA & $58 \%$ & $82 \%$ & $52 \%$ \\
Op. mortality after day 30 & $5 \cdot 5 \%$ & $8 \%$ & $4.9 \%$ \\
Mortality after 1 year & $13 \%$ & $14 \%$ & $8 \%$ \\
\hline
\end{tabular}

Duke data from McNeer (1974) and Rosati (1975); Stanford data from Tecklenberg (1975) and Alderman (1975).

These data are preliminary and further analysis of operative mortality will be made in future reports of the study.

(4) Interpretation of coronary arteriograms. It has now been clearly demonstrated that the prognosis of coronary artery disease is highly dependent on the extent and location of lesions and ventricular function as assessed by angiography. All coronary arteriograms were interpreted by study participants in their local hospitals. Preliminary studies have demonstrated a substantial inter-observer and intraobserver variation in the interpretation of coronary arteriograms (Detre et al., 1975). Other studies have reported observer variations in the interpretation of angiograms (Chaitman et al., 1975). During the course of the study participants developed increasing accuracy and skill in interpretation. For these reasons the arteriograms of all patients in the study have been read a second time by currently active participants. A new, more complete report form has been developed. Second readings using the revised report form were completed on all patients by December 1975.

As an additional check on accuracy of interpretation, all arteriograms will have a third reading by two skilled angiographers who are not participants in the study.

\section{(K) Characteristics of the study population}

A total of 1015 patients have been randomized and entered into the study. Patient entry was discontinued on 31 December 1974 since the number of patients entered was considered sufficient to provide statistically significant data regarding the predetermined end-points of the study.
The patient population in the study cannot be regarded as a random sample of men with stable angina pectoris. The patients were selected by: (1) their eligibility for VA hospital treatment; (2) their admission to a VA clinic or the in-patient service of a participating hospital; (3) possessing the comprehensive inclusion and exclusion characteristics of the protocol; (4) their motivation and willingness to enter the study.

The mean age of all study patients was 50.5 years (range 27-68).

Other baseline characteristics for both treatment groups are summarized in Tables 5-8.

The patient population had a relatively severe degree of coronary artery disease. There was ECG evidence of a prior myocardial infarction in $40 \%$. Over $53 \%$ of patients had a history of angina of longer than 2 years and $56 \%$ were in the $\mathrm{NYH}$ Association functional class III or IV. Prior hypertension, a family history of cardiovascular disease and an elevated serum cholesterol ( $>249 \mathrm{mg} \%$ ) were the most common risk factors occurring in $25 \%, 58 \%$ and $37 \%$ of patients respectively. A systolic blood pressure exceeding $149 \mathrm{~mm}$ and a diastolic pressure exceeding $89 \mathrm{~mm}$ occurred in $13 \%$ and $27 \%$ respectively.

TABLE 5. Baseline variables in medical history

\begin{tabular}{|c|c|c|}
\hline & $\begin{array}{c}\text { Medical } \\
\%\end{array}$ & $\begin{array}{c}\text { Surgical } \\
\%\end{array}$ \\
\hline History of prior infarct & 41 & 39 \\
\hline History of hypertension & $26 \cdot 6$ & $23 \cdot 9$ \\
\hline History of diabetes & $14 \cdot 6$ & $13 \cdot 5$ \\
\hline $\begin{array}{l}\text { Family history of cardiovascular disease } \\
\text { Duration of angina }\end{array}$ & $58 \cdot 4$ & $57 \cdot 9$ \\
\hline 6-12 months & $26 \cdot 5$ & $22 \cdot 2$ \\
\hline 13-24 months & $17 \cdot 9$ & $18 \cdot 4$ \\
\hline 25-60 months & $24 \cdot 8$ & $30 \cdot 2$ \\
\hline$>60$ months & $26 \cdot 9$ & $25 \cdot 2$ \\
\hline N.Y. Heart Association Functional Class & & \\
\hline I & $3 \cdot 1$ & $2 \cdot 3$ \\
\hline II & $38 \cdot 1$ & $36 \cdot 7$ \\
\hline III & $52 \cdot 5$ & $54 \cdot 2$ \\
\hline IV & $2 \cdot 8$ & $2 \cdot 7$ \\
\hline
\end{tabular}

TABLE 6. Objective non-invasive baseline variables

\begin{tabular}{lrr}
\hline & $\begin{array}{c}\text { Medical } \\
(\%)\end{array}$ & $\begin{array}{c}\text { Surgical } \\
(\%)\end{array}$ \\
ECG evidence of prior infarct & $80 \cdot 0$ & $78 \cdot 0$ \\
Systolic blood pressure (mmHg) & & \\
$140-149$ & $16 \cdot 4$ & $16 \cdot 2$ \\
$150-159$ & $6 \cdot 3$ & $6 \cdot 7$ \\
$160-169$ & $4 \cdot 4$ & $2 \cdot 9$ \\
$>170$ & $4 \cdot 4$ & $3 \cdot 1$ \\
Diastolic blood pressure (mmHg) & & \\
$80-89$ & $42 \cdot 0$ & $45 \cdot 7$ \\
$90-99$ & $20 \cdot 6$ & $20 \cdot 0$ \\
$>99$ & $8 \cdot 1$ & $5 \cdot 2$ \\
Serum cholesterol > 249 & $38 \cdot 6$ & $27 \cdot 0$ \\
CT ratio > 49 & $9 \cdot 4$ & $8 \cdot 9$ \\
\hline
\end{tabular}


TABLE 7. Baseline variables from arteriographic studies

\begin{tabular}{lcc}
\hline & $\begin{array}{c}\text { Medical } \\
(\%)\end{array}$ & $\begin{array}{c}\text { Surgical } \\
(\%)\end{array}$ \\
\hline Number of vessels with significant lesions & & \\
One & $16 \cdot 0$ & $15 \cdot 2$ \\
Two & $33 \cdot 1$ & $33 \cdot 1$ \\
Three & $50 \cdot 1$ & $51 \cdot 7$ \\
Contraction abnormalities & $54 \cdot 8$ & $52 \cdot 7$ \\
Ejection fraction $<45$ & $13 \cdot 9$ & $14 \cdot 7$ \\
LVEDP > 14 mmHg & $33 \cdot 7$ & $33 \cdot 3$ \\
Collaterals present & $66 \cdot 7$ & $71 \cdot 7$ \\
Significant left main lesion & $10 \cdot 3$ & 12.6 \\
\hline
\end{tabular}

TABLE 8. Baseline variables of composite risk

\begin{tabular}{lcc}
\hline & $\begin{array}{c}\text { Medical } \\
(\%)\end{array}$ & $\begin{array}{c}\text { Surgical } \\
(\%)\end{array}$ \\
\hline $\begin{array}{l}\text { 1-Vessel disease with normal LV } \\
\text { function }\end{array}$ & 5.9 & 5.5 \\
$\begin{array}{l}\text { 2-Vessel disease with normal LV } \\
\text { function }\end{array}$ & 6.2 & 6.9 \\
$\begin{array}{l}\text { 3-Vessel disease with normal LV } \\
\text { function }\end{array}$ & 10.7 & 11.1 \\
$\begin{array}{l}\text { 1-Vessel disease with abnormal LV } \\
\text { function }\end{array}$ & 8.5 & 8.4 \\
$\begin{array}{l}\text { 2-Vessel disease with abnormal LV } \\
\text { function }\end{array}$ & 20.6 & 21.8 \\
$\begin{array}{l}\text { 3-Vessel disease with abnormal LV } \\
\text { function }\end{array}$ & 36.8 & 37.4 \\
\hline
\end{tabular}

Angiography revealed abnormal LV function, i.e. contraction abnormalities in $54 \%$ of patients. This is comparable to the high incidence of patients with a history of prior infarction $(58 \%)$. It is now known that when ECG signs of prior infarction are present LV contraction abnormalities are usually present (Shettigar et al., 1974).

Resting LV diastolic pressure exceeded $14 \mathrm{mmHg}$ in $34 \%$ of patients and $14 \%$ had ejection fractions of less than $45 \%$.

Fifty-one per cent of patients had 3-vessel coronary disease and $11.5 \%$ had significant left main coronary disease.

The distribution of composite variables is indicated in Table 5.

These objective data also indicate a relatively severe degree of coronary disease in the patient population which is supported by the comparative data in Table 4. In this table, characteristics of the VA study population are compared with the incidence of similar variables in two large series of patients with coronary artery disease who had bypass graft surgery at two non-VA hospital centres.

\section{(L) Comparison of medical and surgical treatment groups}

An important advantage of large scale randomized studies is the equal distribution of patients with similar baseline characteristics into the treatment groups employed. A comparison of the features of patients assigned to medical and surgical therapy indicates no $\stackrel{\odot}{\complement}$ statistically significant difference between the two $c$ groups in terms of their baseline characteristics $\overrightarrow{\vec{c}}$ (Tables 4-7). The objective of randomization has therefore been achieved in the present study.

\section{(M) Results of medical treatment}

A comprehensive analysis of the results of medical versus surgical treatment will be made in future pub- $\nsim$ lications. For illustrative purposes, the cumulative $\vec{\circ}$ survival rates for 516 patients who have been treated medically for 2 years are summarized in Figs 2 and 3 . $\vec{\omega}$ Low risk patients are defined as those who have $\frac{O}{0}$ single vessel disease with or without abnormal LV function and 2- or 3-vessel disease with normal LV if function. High risk patients have 2- or 3-vessel $\mathrm{N}$

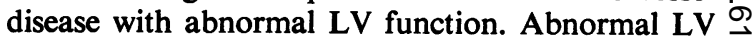
function is defined when at least one of the following three abnormalities is present: (a) LV contraction of abnormality; (b) LV end-diastolic pressure $>140$ mmHg; (c) ejection fraction $<45 \%$. The left main population group includes all medically treated $\vec{\nabla}$ patients with left main lesions regardless of LV $\stackrel{\mathbb{D}}{\circ}$ function.

These data are comparable to those reported i other studies and indicate that survival in stable ang gina is highly dependent on LV function, the pre $\overrightarrow{0}$ sence of left main coronary disease and, to a lesse के

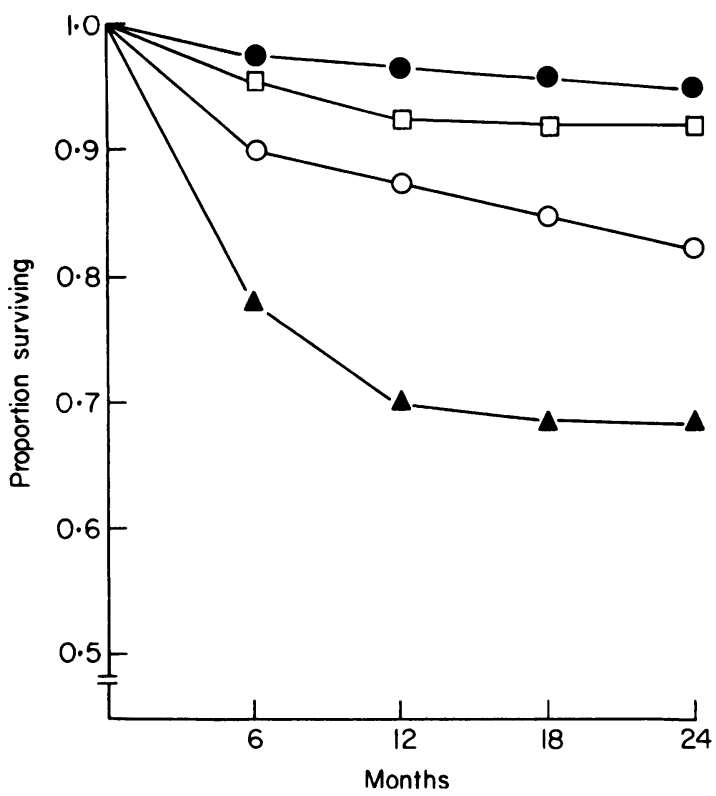

Fig. 2. Cumulative survival rates for medically treated patients. -- Low risk $(n=288) ; \square-\square$, all patients; $\bigcirc-\bigcirc$, high risk $(n=179) ; \Delta-\Lambda$, left main $(n=49)$. 


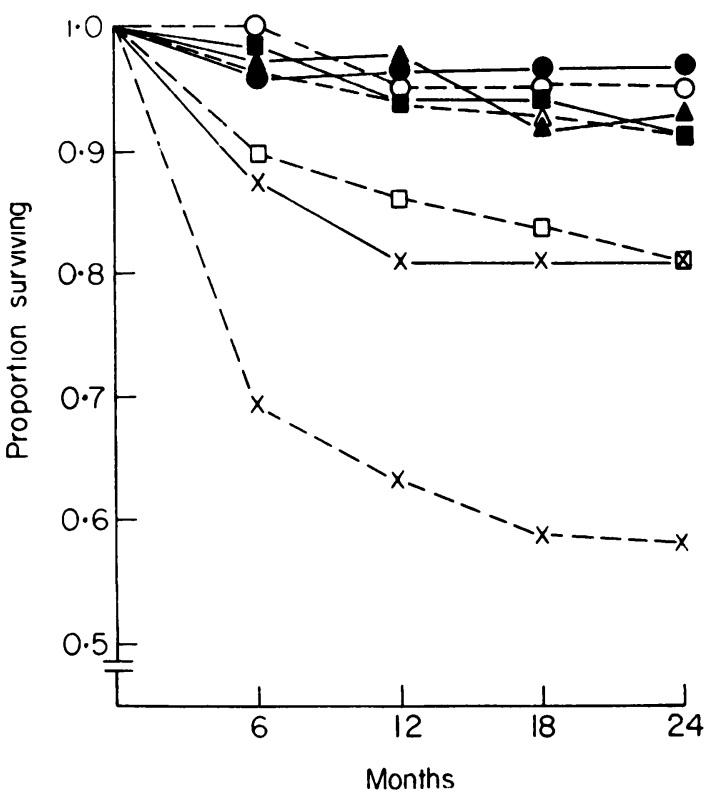

FIG. 3. Cumulative survival rates for medically-treated patients. -O, 1-vessel, normal LVF $(n=23)$; -A, 2-vessel, normal LVF $(n=32)$; $\square$, 3vessel, normal LVF $(n=52)$; $\bigcirc---\bigcirc, 1$-vessel, abnormal LVF $(n=44) ; \triangle \cdots \triangle$, 2-vessel, abnormal LVF $(n=104)$; $\square--\square$, 3-vessel, abnormal LVF $(n=179) ; \times-\times$, left main, normal LVF $(n=18)$; $\times \ldots \times$, left main, abnormal LVF $(n=31)$.

extent, on the number of vessels involved (Burggraf and Parker, 1975).

Survival curves of all groups have a biphasic characteristic, i.e. most deaths occur during the first 6-12 months of observation. There is a common feature of survival curves in any chronic disease state. It suggests that in any defined population group some patients are at higher risk of early death than others. Identification of such high risk patients is an important objective of any study in the natural history of disease. This is being examined in the VA study by the use of multiple, weighted regression equations using definable risk characteristics.

\section{Discussion}

This communication is a report of the current status of a large scale, prospective randomized study and the data presented are the result of preliminary data analyses. Final results will be reported in subsequent publications. Certain aspects of the study deserve comment.

\section{(A) Randomization}

Randomization is a statistical necessity in large scale studies of treatment methods as well as an ethical requirement. In the present study, the statisti- cal identity of the two treatment groups has been achieved. When two methods of therapy are available but the superiority of one method over the other has not been clearly demonstrated, randomization is an ethically acceptable procedure. Randomization ensures that at least $50 \%$ of the patients will receive the proper treatment should it be ultimately shown that one method is superior. In addition, the less effective mode of therapy can be abandoned and thus prevent its continued application over an unduly long period. If appropriate randomized studies of the internal mammary implant operation had been initiated when this procedure was first employed in patients with angina, many unnecessary operations would have been prevented. Appropriate randomized studies are still urgently needed to evaluate other surgical interventions in coronary artery disease such as the value of surgery in acute myocardial infarction (Roberts and Sobel, 1974).

Randomization is not the only method of evaluating surgical versus medical treatment of coronary artery disease. In many hospitals, randomization is not possible owing to various reasons, yet many patients will receive surgical therapy while others will not. If the baseline characteristics of such patients are defined by appropriate methods including angiography, a comparison can be made between therapeutic methods in groups with similar characteristics. Such studies are being carried out in several centres (McNeer et al., 1974; Kouchoukos et al., 1975). The similarity of results obtained by such methods and data currently being obtained in the VA study indicates the value of the data bank method. A limitation of the method is the presence of higher risk factors in medical treatment groups.

Some of the methodological problems of the VA study have been presented in this communication which are common to other similar large scale studies. An important problem is the need for constant surveillance of diagnostic and operative risks so that these can be minimized. Ethical considerations require continuous analysis of the results of the study. In the event that one treatment method is proved to be clearly superior in a given group of patients, further randomization in this group must cease and participants should be informed of the results of the study to permit the patient to choose the preferable therapy if possible. At present it has been demonstrated that in patients with left main coronary disease and poor $\mathrm{LV}$ function survival is improved by surgical treatment (Takaro et al., 1975). Accordingly, all participants and all medical patients in this sub-group have been informed of this observation so that they may elect to have surgical treatment.

This is a preliminary report and comparative mortality for medical and surgical treatment groups 
including sub-groups will be analysed in future publications.

\section{Acknowledgment}

This work was carried out under the auspices of the Veterans Administration Cooperative Study of Surgery for Coronary Arterial Occlusive Disease.

\section{References}

Bhayana, J., Gage, A., Hultgren, H., Detre, K., Takaro, T. \& Participants in the VA Cooperative Study (1975) VA cooperative study of surgery for coronary arterial disease. I. Internal mammary artery implantation. Circulation 52 (Suppl. 2), 161 (abstract).

Burggraf, G. \& Parker, J. (1975) Prognosis in coronary artery disease. Circulation, 51, 146.

Chaitman, B., De Mots, H., Bristow, D., Rasch, J. \& RAHIMtoola, S. (1975) Objective and subjective analysis of left ventricular angiograms. Circulation, 52, 420.

Detre, K.M., Wright, E., MurPhy, M.L. \& Takaro, T. (1975) Observer agreement in evaluating coronary angiograms. Circulation, 52, 979.

EfFler, D.B., Groves, L.K., Sones, F.J., Jr \& Shirey, E.K. (1963) Increased myocardial perfusion by internal mammary implant: Vineberg's operation. Annals of Surgery, 158, 526.

EfFler, D., Sones, F.M., Jr, Groves, L. \& Suarez, E. (1965) Myocardial revascularization by Vineberg's internal mammary implant: evaluation of postoperative results. Journal of Thoracic and Cardiovascular Surgery, 50, 527.

Hultgren, H. \& Hurley, E. (1968) Surgery in obstructive coronary artery disease. Advances in Internal Medicine, 14, 107.
Kouchoukos, N., Oberman, A., Russell, R. \& Jones, W. (1975) Surgical versus medical treatment of occlusive $\mathbb{D}$ disease confined to the left anterior descending coronary artery. American Journal of Cardiology, 35, 836.

McNeer, J., Starmer, F., Bartel, A., Behar, V., Kong, Y., Peter, R. \& Rosati, R. (1974) The nature of treatment selection in coronary artery disease. Experience with medical and surgical treatment of a chronic disease. Circulation, 49, 606

Pfeifer, J., Hultgren, H. \& Fails, H. (1974) Evaluation of a questionnaire to determine severity of angina pectoris. Clinical Research, 22, 153A (abstr.).

ROBERTS, R. \& Sobel, B. (1974) Coronary revascularization during evolving myocardial infarction-the need for ? caution. Circulation, 50, 867.

Selzer A., Anderson, W. \& March, H. (1971) Indications for coronary arteriography-risks versus benefits. California Medicine, 115, 1.

Shettigar, U., Hultgren, H., Pfeifer, J. \& Lipton, M. 3 (1974) Diagnostic value of $Q$ waves in inferior myocardial iv infarction. American Heart Journal, 88, 170.

Takaro, T., Hultgren, H., Detre, K. \& Participants in the VA Cooperative Study (1975) VA cooperative study of coronary arterial surgery. II. Left main disease. Circula- जे tion, 52 (Suppl. 2), 143 (abstr.).

Takaro, R. Hultgren, H., Littman, D. \& Wright, E. 윽 (1973) An analysis of deaths occurring in association with $\rightarrow$ coronary arteriography. American Heart Journal, 86, 587.

ViNEBERG, A. (1964) Experimental background of myocardial revascularization by internal mammary implantation and supplementary technics with its clinical application in 125 patients. A review and critical appraisal. Annals of Surgery 159, 185 